\title{
A PARACOMPACT SEMI-METRIC SPACE WHICH IS NOT AN $M_{3}$-SPACE ${ }^{1}$
}

\section{R. W. HEATH}

E. A. Michael has shown that a regular Hausdorff space $S$ is paracompact if and only if any of the following is true: (1) every open cover of $S$ has a $\sigma$-locally finite open refinement [8], (2) every open cover of $S$ has an open $\sigma$-closure preserving refinement [9], or (3) every open cover of $S$ has an open $\sigma$-cushioned refinement [10]. The Nagata-Smirnov Theorem [12] or [14] (see also Bing's Theorem 3 in [1]) states that a $T_{3}$-space with a $\sigma$-locally finite base is metrizable.

In [3] Jack Ceder defines an $M_{1}$-space to be a $T_{3}$-space with a $\sigma$-closure preserving base and an $M_{3}$-space to be a $T_{1}$-space with a $\sigma$-cushioned pair base (see Definition 1 below). By Michael's theorems cited above both $M_{1}$ - and $M_{3}$-spaces are paracompact, and in view of the Nagata-Smirnov Theorem, one might suspect that they would even be metrizable. In [3], however, Ceder shows that such is not the case; in fact $M_{1}$ - and $M_{3}$-spaces need not be first countable, and they need not be metrizable even if they are first countable. Ceder does show, though, that a first countable $M_{3^{-}}$(and hence $M_{1^{-}}$) space is a paracompact semi-metric space, and he raises the question: is this a characterization-i.e. is every paracompact semi-metric space an $M_{3}$-space (and perhaps even an $M_{1}$-space)? A negative answer is given to that question in this paper.

Also it was called to my attention by Professor Michael that a question raised by $C$. Borges [2] is answered (in the negative) by the same example below, which is a cosmic space (the regular continuous image of a separable metric space [11]) that is not an $M_{3}$-space.

DEFINITION 1. Let $P$ be a collection of ordered pairs of subsets of the $T_{1}$-space $S$ such that, for each $p=\left(p_{1}, p_{2}\right) \in P, p_{1}$ is open and $p_{1} \subset p_{2}$, and such that, for every $x \in S$ and every neighborhood $U$ of $x$, there is a $p \in P$ for which $x \in p_{1} \subset p_{2} \subset U$. Then $P$ is called a pair base for $S$. Moreover, $P$ is called cushioned if, for every $Q \subset P$,

$$
\mathrm{Cl} \cup\left\{p_{1}: p \in Q\right\} \subset \cup\left\{p_{2}: p \in Q\right\}
$$

and $P$ is $\sigma$-cushioned if it is the union of countably many cushioned collections.

An $M_{3}$-space is a $T_{1}$-space with a $\sigma$-cushioned pair base.

Presented to the Society December 29, 1965 ; received by the editors June 19, 1965.

1 The research for this paper was partially suppor ted by National Science Foundation grant NSF G-23790. 
Note that Borges calls $M_{3}$-spaces stratifiable spaces in [2]. Also, first countable $M_{3}$-spaces are sometimes called Nagata spaces [3].

Definition 2. A $T_{1}$-space $S$ is a semi-metric space provided that there is a function $d$ from $S \times S$ into the nonnegative reals such that

(1) for every $(x, y) \in S \times S, d(x, y)=d(y, x)$ and $d(x, y)=0$ if and only if $x=y$ and

(2) for every $x \in S$ and $M \subset S$, inf $\{d(x, y): y \in M\}=0$ if and only if $x \in \mathrm{Cl} M$ (i.e., "the topology is invariant with respect to $d$ ").

All other terms are defined as in [7] or [13].

Alternative characterization of semi-metric spaces (Theorem 3.2 of [4]: A $T_{1}$-space $S$ is semi-metric if and only if there is a collection $\{g(n, x): x \in S, n=1,2, \cdots\}$ of open sets such that (1) for each $x \in S,\{g(n, x): n=1,2, \cdots\}$ is a local base for the topology at $x$ and (2) if $y \in S$ and $x$ is a point sequence such that, for each $m, y$ $\in g\left(m, x_{m}\right)$, then $x$ converges to $y$.

THEOREM 1. There exists a regular Lindelöf (and hence paracompact) semi-metric space $S$ which is a cosmic space (the continuous image of a separable metric space) but is not an $M_{3}$-space.

Proof. Such a space $S$ is defined as follows. The points of $S$ are those points $z$ of the complex plane such that either (1) $\operatorname{Im} z=0$ and $\operatorname{Re} z$ is irrational or (2) $\operatorname{Im} z>0$ and both $\operatorname{Im} z$ and $\operatorname{Re} z$ are rational. For each point $z \in S$ of type 1 (i.e., $\operatorname{Im} z=0$ ) and each natural number $n, B(n, z)=\{x \in S: \operatorname{Im} x<|\operatorname{Re}(x-z)|<1 / n$ or $z=x\}$ (i.e. the "bowtie region" of radius $1 / n$ and vertex angle $45^{\circ}$ ) is a basis element for $S$; and for each $z \in S$ of type 2 and for each $n$, the open disc of radius $1 / n$ and center $z$ is a basis element. The space is clearly regular since, for every $z \in S$ with $\operatorname{Im} z=0$ and for each $n, B(n, z)$ has only the two points $z+1 / n$ and $z-1 / n$ of $S$ on its boundary (all points of its boundary in the complex plane having at least one coordinate irrational). Also $S$ is easily seen to be semi-metric by the above alternative characterization, and $S$ is clearly Lindelöf. That $S$ is a cosmic space follows easily by the same argument given for Example 12.1 in [11]. Finally, $S$ is not an $M_{3}$-space. For suppose that there were a $\sigma$-cushioned pair base $P=\bigcup\{Q(i): 1=1,2, \cdots\}$ for $S$. Then there would be a second category subset $M$ of the $x$-axis, with $M \subset S$, and natural numbers $m$ and $k$ such that, for each $x \in M$, there is a $q \in Q(k)$ such that

$$
B(m, x) \subset q_{1} \subset q_{2} \subset B(1, x) .
$$

Pick a rational number $r$ in the closure (with respect to the Euclidean topology) of $M$. Then $r$ is in the closure of $\{x \in M: x>r\}$ or of 
$\{x \in M: x<r\}$; assume the former. Let $R \subset Q(k)$ consist of all $q \in Q(k)$ which satisfy $(*)$ for some $x \in M$ with $r<x<r+1 / m$. Then, for $y=(r+1 / m, 1 / m), y \in \mathrm{Cl} \cup\left\{p_{1}: p \in R\right\}$ but $y \in \cup\left\{p_{2}: p \in R\right\}$. That contradicts the assumption that $Q(k)$ is cushioned. Thus $S$ is not an $M_{3}$-space.

The questions remain (1) whether every $M_{3}$-space is an $M_{1}$-space (see [3] for the definition of an $M_{2}$-space, an "intermediate" space), (2) what topological condition is necessary in order for a paracompact semi-metric space to be an $M_{3}$-space, (3) whether every Lindelöf semi-metric space is a cosmic space, (4) whether every separable $M_{3^{-}}$ space is a cosmic space (see [2]) and (5) whether every regular countable space is an $M_{3}$-space. For some theorems relating semimetric and $M_{3}$-spaces see [5], and for a necessary and sufficient condition that an $M_{3}$-space be metrizable (namely: that it have a pointcountable base) see [6].

\section{REFERENCES}

1. R. H. Bing, Metrization of topological spaces, Canad. J. of Math. 3 (1951), $175-186$.

2. C. J. R. Borges, On stratifiable spaces, Pacific J. Math. 17 (1966), 1-16.

3. Jack Ceder, Some generalizations of metric spaces, Pacific J. Math. 11 (1961), $105-125$.

4. R. W. Heath, Arc-wise connectedness in semi-metric spaces, Pacific J. Math. 12 (1962), 1301-1319.

5. - On open mappings and certain spaces satisfying the first countability axiom, Fund. Math. 57 (1965), 91-96.

6. - On spaces with point-countable bases, Bull. Acad. Polon. Sci. Sér. Sci. Math. Astronom. Phys. 13 (1965), 393-395.

7. J. L. Kelley, General topology, Van Nostrand, Princeton, N. J., 1955.

8. E. A. Michael, $A$ note on paracompact spaces, Proc. Amer. Math. Soc. 4 (1953), $831-838$

9. - Another note on paracompact spaces, Proc. Amer. Math. Soc. 8 (1957), 822-828.

10. - Yet another note on paracompact spaces, Proc. Amer. Math. Soc. 10 (1959), 309-314.

11. - $\aleph_{0}$-spaces, J. Math. Mech. (to appear).

12. J. Nagata, On a necessary and sufficient condition of metrizability, J. Inst. Polytech. Osaka City Univ. 1 (1950), 93-100.

13. R. L. Moore, Foundations of point set theory, rev. ed., Amer. Math. Soc. Colloq. Publ. Vol. 13, Providence, R. I., 1962.

14. Yu. M. Smirnov, A necessary and sufficient condition for metrizability of a topological space, Dokl. Acad. Nauk SSSR 77 (1951), 197-200.

Arizona State University and

UNIVERSITY OF GEORGIA 